\title{
Growth temperature and V/III ratio effects on the morphology and crystal structure of InP nanowires.
}

\begin{abstract}
The effects of growth temperature and V/III ratio on the morphology and crystallographic phases of InP nanowires that are grown by metal organic chemical vapour deposition have been studied. We show that higher growth temperatures or higher V/III ratios promote the formation of wurtzite nanowires while zinc-blende nanowires are favourable at lower growth temperatures and lower V/III ratios. A schematic map of distribution of zinc-blende and wurtzite structures has been developed in the range of growth temperatures $\left(400-510{ }^{\circ} \mathrm{C}\right)$ and V/III ratios (44 to 700) investigated in this study.
\end{abstract}

Keyword: Nanowires; InP; MOCVD; Temperature; V/III ratio; Crystal structure. 